\title{
Desordens hematológicas em onças-pardas (Puma concolor; Linnaeus, 1771) infectadas por Citauxzoon felis
}

[Hematological disorders in Brown jaguars (Puma concolor; Linnaeus 1771) Infected by Citauxzoon felis $]$

P.M.P. Silva ${ }^{1}$, A.S. Rezende ${ }^{1}$, G.G. Oliveira ${ }^{1}$, K.C.S. Godoy ${ }^{1}$, T.R. Antunes ${ }^{1}$, M.C.C. Silva ${ }^{1}$, L.B.S. Azuaga ${ }^{2}$, C.A.N. Ramos ${ }^{3}$, A.I. Souza ${ }^{3}$

\author{
${ }^{1}$ Aluno de pós-graduação - Faculdade de Medicina Veterinária e Zootecnia - Universidade \\ Federal de Mato Grosso do Sul - Campo Grande, MS \\ ${ }^{2}$ Centro de Reabilitação de Animais Silvestres - Campo Grande, MS \\ ${ }^{3}$ Faculdade de Medicina Veterinária e Zootecnia - Universidade \\ Federal de Mato Grosso do Sul - Campo Grande, MS
}

\begin{abstract}
RESUMO
Este estudo objetivou descrever o aspecto hematológico de seis onças-pardas (Puma concolor) infectadas pelo Cytauxzoon felis. Os seis casos de infecção foram identificados durante o manejo sanitário de 11 animais de um centro de reabilitação de animais silvestres. Estruturas compatíveis com piroplasmídeos foram observadas durante a avaliação do esfregaço sanguíneo e confirmadas como Cytauxzoon felis pela técnica de PCR. A análise estatística demonstrou diferença significativa $(\mathrm{P}<0,05)$ no número absoluto dos linfócitos entre os grupos dos animais infectados e não infectados. Assim, expressivas alterações hematológicas e bioquímicas entre os grupos investigados alertam para a dificuldade de identificação de onças-pardas infectadas por $C$. felis, apoiada apenas em exames de rotina, bem como para o risco, sobretudo, da reintrodução desses animais na natureza.
\end{abstract}

Palavras-chave: cytauxzoonose, diagnóstico, felino selvagem

\begin{abstract}
This Cytauxzoon felis by the PCR technique. Statistical analysis showed a significant difference is study aimed to describe the hematological appearance of six puma (puma concolor) infected with cytauxzoon felis. The six cases of infection were identified during the sanitary management of 11 animals from a wild animal rehabilitation center. Piroplasmid compatible structures were observed during the blood smear evaluation and confirmed as $(P<0.05)$ in the absolute number of lymphocytes between the groups of infected and uninfected animals. Thus expressive hematological and biochemical alterations between the groups investigated alert to the difficulty of identifying infected brown jaguars by $\mathrm{C}$. felis, supported only by routine examinations, and the risk especially when aiming at the reintroduction of these animals in the wild.
\end{abstract}

Keywords: cytauxzoonose, diagnosis, wild feline

\section{INTRODUÇÃO}

Cytauxzoon felis é um piroplasmídeo da família Theileriidae que infecta felídeos (Pesquera et al., 2015). O agente é vetorialmente transmitido durante o repasto sanguíneo de carrapatos infectados da família Ixodidae. No hospedeiro vertebrado, localiza-se preferencialmente em eritrócitos, mas já foi observado em diferentes tecidos (Garner et al., 1996).

Os felinos selvagens são considerados reservatórios do parasito (Butt et al., 1991), embora casos de óbitos já tenham sido atribuídos

Recebido em 9 de setembro de 2018

Aceito em 1 de outubro de 2019

E-mail: polyana_mayume@hotmail.com 
ao agente (Jakob e Wesemeier, 1996; Garner et al., 1996; Peixoto et al., 2007). Apesar disso, a caracterização laboratorial de felinos selvagens infectados pelo Cytauxzoon felis foi raramente descrita (Fowler e Miller, 1986). O conhecimento dessas alterações pode auxiliar na triagem e identificação precoce desses indivíduos, assim como no controle da transmissão do agente, especialmente dos animais em cativeiro, candidatos à reintrodução na natureza.

Diante disso, o objetivo deste estudo foi verificar a existência de alterações hematológicas e bioquímicas decorrentes da infecção de Cytauxzoon felis em onças-pardas (Puma concolor) mantidas em cativeiro na região central do Brasil.

\section{MATERIAL E MÉTODOS}

Amostras sanguíneas de 11 onças-pardas foram coletadas durante o manejo sanitário de rotina, realizado no Centro de Reabilitação de Animais Silvestres (Cras), na cidade de Campo Grande (latitude $-20^{\circ} 26^{\prime} 34^{\prime \prime} \mathrm{S}$, longitude $-54^{\circ} 38^{\prime} 47^{\prime \prime} \mathrm{W}$ ), Mato Grosso do Sul, Brasil.

Para a coleta do material, os animais foram sedados com medetomidina e quetamina, as amostras de sangue obtidas por venopunção e acondicionadas em tubos contendo EDTA (ácido etilenodiaminotetra-acético), para realização do hemograma, e em tubos sem anticoagulantes, para a obtenção do soro, que, após coagulação e centrifugação, foi armazenado em microtubo a $-80^{\circ} \mathrm{C}$ até o momento das análises bioquímicas.

Os hemogramas foram processados em analisador hematológico automatizado Sysmex ${ }^{\circledR}$ pocH$100 \mathrm{iV}$. As concentrações séricas de albumina, globulina, cálcio total, fósforo, colesterol total, creatinina, proteína total, ureia e atividade das enzimas alanina aminotransferase (ALT), aspartatoaminotransferase (AST) e creatinina quinase foram analisadas em equipamentos automatizados (Cobas $\left.\mathrm{C} 111^{\circledR}\right)$.

Todas as amostras foram submetidas à pesquisa de hemoparasita no esfregaço sanguíneo e à investigação para Babesia sp., Theileria sp. e Cytauxzoon sp., por meio de reação em cadeia da polimerase (PCR), utilizando-se o protocolo descrito por Brown et al. (2010). A extração de DNA foi realizada a partir de $350 \mu \mathrm{L}$ de sangue periférico, mediante metodologia descrita por Araújo et al. (2009). A quantidade e a integridade das amostras extraídas foram avaliadas por espectrofotometria em aparelho BioPhotometer Plus (Eppendorf) e eletroforese em gel de agarose $0,8 \%$, respectivamente. As amostras de DNA extraídas foram submetidas à reação de PCR com os primers BT1-F e BT1-R, previamente desenvolvidos por Criado-Fornelioet al. (2003) para amplificar uma região do gene $18 \mathrm{~S}$ rRNA (aproximadamente $400 \mathrm{pb}$ ) comum aos gêneros Babesia e Theileria.

Dois grupos de animais foram constituídos mediante presença ou ausência da infecção por Cytauxzoon felis. Os parâmetros hematológicos e bioquímicos entre os grupos foram comparados pelo teste $\mathrm{t}$ de Student. O valor de $\mathrm{P}$ foi considerado significativo quando $<0,05$.

\section{RESULTADOS}

Das 11 amostras de sangue avaliadas, seis apresentavam estruturas compatíveis com piroplasmídeos em esfregaço sanguíneo, que foram confirmadas como Cytauxzoon felis pela técnica de PCR. As amplificações do DNA das amostras resultaram em fragmentos com aproximadamente $600 \mathrm{pb}$ em seis animais. Após sequenciamento dos fragmentos amplificados, obtiveram-se sequências de consenso de aproximadamente 532pb. $\mathrm{Na}$ busca por homologia com sequências de DNA disponíveis no Genbank, observou-se identidade de $98 \%$ com sequências do gene 18S rRNA de Cytauxzoon felis em todas as amostras.

Não se observou identidade significativa com outros protozoários. Os parâmetros hematológicos dos animais infectados $(n=6)$ e não infectados $(n=5)$ são apresentados na Tab. 1. O número absoluto de linfócitos apresentou diferença estatística significativa quando os grupos de animais infectados e não infectados foram comparados $(\mathrm{P}=0,007)$. Os parâmetros bioquímicos dos animais infectados $(n=6)$ e não infectados ( $\mathrm{n}=5)$ são apresentados na Tab. 2. 
Tabela 1. Média e desvio-padrão dos parâmetros hematológicos de Puma concolor (onça-parda) infectado e não infectado por Cytauxzoon felis do Centro de Reabilitação de Animais Silvestres (Cras) de Campo Grande, Mato Grosso do Sul, Brasil

\begin{tabular}{llll}
\hline Parâmetros & Onças infectadas & Onças não infectadas & P-vale \\
\hline Eritrócitos $\left(x 10^{6} / \mu \mathrm{L}\right)$ & $8,23^{\mathrm{a}} \pm 1,50$ & $9,25^{\mathrm{a}} \pm 1,16$ & 0,24 \\
Hemoglobina $(\mathrm{g} / \mathrm{dL})$ & $11,88^{\mathrm{a}} \pm 1,64$ & $12,96^{\mathrm{a}} \pm 1,92$ & 0,34 \\
Volume globular $(\%)$ & $34,53^{\mathrm{a}} \pm 3,95$ & $37,64^{\mathrm{a}} \pm 5,16$ & 0,28 \\
VCM $(\mathrm{fL})$ & $42,78^{\mathrm{a}} \pm 2,53$ & $40,68^{\mathrm{a}} \pm 2,36$ & 0,19 \\
CHCM $(\mathrm{g} / \mathrm{dL})$ & $34,37^{\mathrm{a}} \pm 2,03$ & $34,40^{\mathrm{a}} \pm 1,15$ & 0,97 \\
RDW-SD $(\mathrm{fL})$ & $33,27^{\mathrm{a}} \pm 3,05$ & $31,48^{\mathrm{a}} \pm 0,76$ & 0,21 \\
RDW-CV $(\%)$ & $15,70^{\mathrm{a}} \pm 0,74$ & $15,86^{\mathrm{a}} \pm 1,05$ & 0,46 \\
Leucócitos $(/ \mu \mathrm{L})$ & $5750^{\mathrm{a}} \pm 1457$ & $6940^{\mathrm{a}} \pm 1439$ & 0,20 \\
Bastonetes $(/ \mu \mathrm{L})$ & $31,00^{\mathrm{a}} \pm 50,00$ & $29,00^{\mathrm{a}} \pm 42,00$ & 1,00 \\
Segmentados $(/ \mu \mathrm{L})$ & $3864^{\mathrm{a}} \pm 1314$ & $4044,00^{\mathrm{a}} \pm 1163,00$ & 0,81 \\
Linfócitos $(/ \mu \mathrm{L})$ & $1502,00^{\mathrm{a}} \pm 481,00$ & $2626,00^{\mathrm{b}} \pm 561,00$ & 0,006 \\
Monócitos $(/ \mu \mathrm{L})$ & $201,00^{\mathrm{a}} \pm 100,00$ & $150,00^{\mathrm{a}} \pm 65,00$ & 0,33 \\
Eosinófilos $(/ \mu \mathrm{L})$ & $153,00^{\mathrm{a}} \pm 81,00$ & $90,00^{\mathrm{a}} \pm 103,00$ & 0,14 \\
Plaquetas $\left(x 10^{3} / \mu \mathrm{L}\right)$ & $278,00^{\mathrm{a}} \pm 124,9$ & $285,60^{\mathrm{a}} \pm 94,13$ & 0,92 \\
\hline
\end{tabular}

VCM: volume corpuscular médio; CHCM: concentração de hemoglobina corpuscular média; RDW: Red Cell Distribution Width. Médias seguidas de letras minúsculas iguais não diferem estatisticamente pelo teste t de Student $(\mathrm{P}<0,05)$

Tabela 2. Média e desvio-padrão dos bioquímicos séricos de Puma concolor (onça-parda) infectado e não infectado por Cytauxzoon felis do Centro de Reabilitação de Animais Silvestres (Cras) de Campo Grande, Mato Grosso do Sul, Brasil

\begin{tabular}{llcl}
\hline Parâmetros & Onças infectadas & Onças não infectadas & P-vale \\
\hline ALT (U/L) & $42,8^{\mathrm{a}} \pm 14,81$ & $38,96^{\mathrm{a}} \pm 14,83$ & 0,67 \\
AST (U/L) & $35,63^{\mathrm{a}} \pm 13,78$ & $39,44^{\mathrm{a}} \pm 19,48$ & 0,71 \\
Albumina $(\mathrm{g} / \mathrm{dL})$ & $4,48^{\mathrm{a}} \pm 0,48$ & $4,28^{\mathrm{a}} \pm 0,44$ & 0,48 \\
Cálcio total (mg/dL) & $10,67^{\mathrm{a}} \pm 0,57$ & $10,15^{\mathrm{a}} \pm 0,32$ & 0,10 \\
Creatinina (mg/dL) & $2,38^{\mathrm{a}} \pm 0,68$ & $2,40^{\mathrm{a}} \pm 0,44$ & 0,91 \\
Fósforo (mg/dL) & $5,13^{\mathrm{a}} \pm 0,53$ & $5,12^{\mathrm{a}} \pm 0,50$ & 0,99 \\
PT (g/dL) & $6,82^{\mathrm{a}} \pm 0,5$ & $6,38^{\mathrm{a}} \pm 0,9$ & 0,31 \\
Globulina (g/dL) & $2,34^{\mathrm{a}} \pm 0,8$ & $2,10^{\mathrm{a}} \pm 1,13$ & 0,71 \\
Uréia $(\mathrm{mg} / \mathrm{dL})$ & $60,21^{\mathrm{a}} \pm 14,46$ & $61,28^{\mathrm{a}} \pm 9.0$ & 0,89 \\
\hline
\end{tabular}

ALT: alanina aminotransferase; AST: aspartatoaminotransferase; PT: proteínas totais séricas. Médias seguidas de letras minúsculas iguais não diferem estatisticamente pelo teste t de Student $(\mathrm{P}<0,05)$.

\section{DISCUSSÃO}

Citauxzoonose é uma doença de felídeos, transmitida por carrapatos, causada pelo protozoário Cytauxzoon felis. Em gatos domésticos (Felis catus), caracteriza-se por apresentar evolução aguda e, geralmente, fatal (Wagner et al., 1980). Ao contrário, em felinos selvagens, a maioria dos casos relatados corrobora a ausência de sinais clínicos observada nos animais, comprovadamente infectados, do presente trabalho (Soares et al., 2004; André et al., 2009). A infecção subclínica pode indicar eficácia imunológica no controle do patógeno, bem como o fato de esses animais poderem atuar como reservatório natural da doença, assim como 
o Lynx rufus (Shock et al.,2012), mas não se descarta o risco de transmissão da doença para outras espécies de felinos selvagens nos casos de reintrodução dessas onças ao seu habitat natural.

O índice de infecção nos animais avaliados foi inferior ao identificado por Furtado et al. (2017) em Panthera onca de vida livre, mas superior às investigações realizadas em diferentes espécies de felídeos mantidos em cativeiro no Brasil (André et al., 2009; Filoni et al., 2012). Essa diferença pode estar relacionada às distintas condições de vida dos animais (vida livre x cativeiro), à estação do ano e densidade e grau de exposição ao vetor (Shock et al.,2012).

Os organismos do gênero Cytauxzoon existem em duas fases distintas: uma eritrocitária e uma tecidual. Infecção experimental demonstrou que apenas os animais que desenvolvem a fase tecidual apresentam manifestações clínicas da doença. Animais que conseguem sobreviver e eliminar a fase de esquizogonia permanecem infectados cronicamente com o estágio intraeritrocitário do agente (Cowell et al., 1988; Nietfeld e Pollock, 2002).

Manifestações laboratoriais de cytauxzoonose em felinos domésticos costumam demonstrar quadros graves de citopenias associados à hiperbilirrubinemia e à hipoalbuminemia (Meinkoth e Kocan, 2005). Felinos da espécie Puma concolor couguar apresentaram anemia, trombocitopenia, bilirrubinemia, hiperproteinemia e aumento da atividade sérica das enzimas ALT e AST sem sinal clínico de doença, três semanas após transferência para área endêmica para o $C$. felis, e os achados foram atribuídos à infecção aguda pelo agente (Harvey et al., 2007).

Dos parâmetros avaliados no presente trabalho, apenas o número de linfócitos foi significativamente menor no grupo dos animais infectados e pode estar associado ao estresse da contenção (Nogueira e Silva, 1997). O cortisol, liberado em quadros de estresse, reduz a recirculação de linfócitos e consequentemente a presença dessas células no sangue periférico (Stockham e Scott, 2014). Vale ressaltar, porém, que são animais mantidos em cativeiro há vários meses e que, portanto, o período pré-patente da infecção não foi determinado, não se descartando a possibilidade de que mudanças possam ter ocorrido anteriormente (Rotstein et al., 1999).

A ausência de alterações clínicas e laboratoriais nas onças-pardas estudadas indica que a infecção crônica pelo $C$. felis não tem efeito negativo sobre esses animais, entretanto cabe considerar que os efeitos do $C$. felis em muitas espécies de felinos selvagens ainda são desconhecidos e que a reintrodução desses animais em seus habitats naturais deve ser cautelosa.

\section{REFERÊNCIAS}

ANDRÉ, M.R.; ADANIA, C.H.; MACHADO, R.Z.; ALLEGRETTI, S.M. et al. Molecular Detection of Cytauxzoon spp. in asymptomatic Brazilian wild captive felids. J. Wildl. Dis., v.45, p.234-237, 2009.

ARAUJO, F.R.; RAMOS, C.A.N.; LUÍZ, H.L.; PÉRES, I.A.H.F.S. et al. Avaliação de um protocolo de extração de DNA genômico a partir de sangue total. Campo Grande: Embrapa Gado de Corte, 2009. 5p. (Comunicado Técnico, n.120).

BROWN, H.M.; LOCKHART, J.M.; LATIMER, K.S.; PETERSON, D.S. Identification and genetic characterization of Cytauxzoon felis in assymptomatic domestic cats and bob cats. Vet. Parasitol., v.172, p.311-316, 2010.

BUTT, M.T.; BOWMAN, D.; BARRM. C.; ROELKE, M.E. Iatrogenic transmission of Cytauxzoon felis from a Florida panther to a domestic cat. J. Wildl. Dis., v.27, p.342-355, 1991.

COWELL, R.L.; FOX, J.C.; PANCIERA, R.J.; TYLER, R.D. Detection of anticytauxzoon antibodies in cats infected with a Cytauxzoon organism from bobcats. Vet. Parasitol., v.28, p.43-52, 1988

CRIADO-FORNELIO, A.; MARTINEZMARCOS, A.; BULING-SARANA, A.; BARBA-CARRETERO, J.C. Molecular studies on Babesia, Theileria and Hepatozoon in southern Europe: part I. Epizootiological aspects. Vet. Parasitol., v.113, p.189-201, 2003.

FILONI, C.; CATÃO-DIAS, J.L.; CATTORI, V.; WILLI, B. et al. Surveillance using serological and molecular methods for the detection of infectious agents in captive Brazilian neotropic and exotic felids. J. Vet. Diag. Invest., v.24, p.166-173, 2012. 
FOWLER, M.E.; MILLER, R.E. Zoo and wild animal medicine: current therapy. San Diego: Elsevier Health Sciences, 1986. cap. 48, p.800807.

FURTADO, M.M.; TANIWAKI, S.A.; METZGER, B.; SANTOS, K.P. et al. Is the freeranging jaguar (Pantheraonca) a reservoir for Cytauxzoonfelis in Brazil?. Ticks Tick Borne Dis., v.8, p.470-476, 2017.

GARNER, M.M.; LUNG, N.P.; CITINO, S.; GREINER, E.C. et al. Fatal cytauxzoonosis in a captive-reared white tiger (Pantheratigris). Vet. Pathol., v.33, p.82-86, 1996.

HARVEY, J.W.; DUNBAR, M.R.; NORTON, T.M.; YABSLEY, M.J. Laboratory findings in acute Cytauxzoonfelis infection in cougars (Puma concolorcouguar) in Florida. J. Zoo Wildl. Med., v.38, p.285-292, 2007.

JAKOB, W.; WESEMEIER, H.H. A Fatal infection in a Bengal tiger resembling Cytauxzoonosis in domestic cats. J. Comp. Pathol., v.114, p.439-444, 1996. MEINKOTH, J.H.; KOCAN, A.A. Feline cytauxzoonosis. Vet. Clin. Small Anim. Pract., v.35, p.89-101, 2005.

NIETFELD, J.C.; POLLOCK, C. Fatal cytauxzoonosis in a free-ranging bob cat (Lynx rufus). J. Wildl. Dis., v.38, p.607-610, 2002.

NOGUEIRA， G.P.; SILVA， J.C.R. Plasma cortisol levels in captive wild felines after chemical restraint. Braz. J. Med. Biol. Res., v.30, p.1359-1361, 1997.
PEIXOTO, P.V.; SOARES, C.O.; SCOFIELD, A.; SANTIAGO C.D. et al. Fatal cytauxzoonosis in captive-reared lions in Brazil. Vet. Parasitol., v.145, p.383-387, 2007.

PESQUERA C.; PALOMAR A.M.; PORTILLO A.; VENZAL J.M. OTEO J.A. Investigation of tick-borne bacteria (Rickettsia spp., Anaplasmaspp., Ehrlichia spp. and Borrelia spp.) in ticks collected from Andean tapirs, cattle and vegetation from a protected area in Ecuador. Parasit. Vectors, v.8, p.46, 2015.

ROTSTEIN, D.S.; TAYLOR, S.K.; HARVEY, J.W.; BEAN, J. Hematologic effects of cytauxzoonosis in Florida panthers and Texas cougars in Florida. J. Wildl. Dis., v.35, p.613-617, 1999.

SHOCK, B.C.; BIRKENHEUER, A.J.; PATTON, L.L.; OLFENBUTTEL, C. et al. Variation in the ITS-1 and ITS-2 rRNA genomic regions of Cytauxzoonfelis from bobcats and pumas in the eastern United States and comparison with sequences from domestic cats. Vet. Parasitol., v.190, p.29-35, 2012.

SOARES, R.J.; SOUZA, A.I.; NETTO, N.T.; SCHEIDE, R.; SCOFIELD, A. Cytauxzoonfelislike in the Moutain Lion (Puma concolor): a case report. J. Anim. Vet. Adv., v.3, p.820-823, 2004.

STOCKHAM, S.L.; SCOTT, M.A. Fundamentos de patologia clínica veterinária. 2.ed. Rio de Janeiro: Guanabara Koogan, 2014. 760p.

WAGNER, J.E.; FERRIS, D.H.; KIER, A.B.; WIGHTMAN, S.R. et al. Experimentally induced cytauxzoonosis-like disease in domestic cats. Vet. Parasitol., v.6, p.305-311 1980. 